\title{
Sinn und Unsinn von Untersuchungen in der Schwangerenvorsorge
}

\author{
Dr. Katharina Lüdemann, Delmenhorst
}

Seit 50 Jahren gibt es ärztliche Schwangerenvorsorge in Deutschland. Die Zahl der Untersuchungen ist stetig gestiegen. Viele Untersuchungen sind weder evidenzbasiert noch hilfreich. Sie verunsichern statt zu beruhigen und liefern zweifelhafte Ergebnisse. Warum führt das geniale Konzept, durch Vorsorge rechtzeitig die Weichen zu stellen für die Gesundheit von Mutter und Kind, so oft zu bescheidenen Ergebnissen? Was läuft schief in der Schwangerenvorsorge?

\section{Fallbeispiel}

Freitag, 16.30 Uhr, in einer deutschen Geburtsklinik.

Frau H. steht vor dem Kreißsaal mit Koffer und Einweisung: „1. Kind. 38. SSW, Ödeme, Makrosomie, zur Einleitung“. Nach Anmeldung, Aufnahme-CTG und Blutentnahme kommt Frau H. zum Ultraschall. Mir fällt ihr riesiger Bauch auf. Ich bitte sie, sich hinzulegen und taste den Bauch ab. Groß, prall, das Fruchtwasser schwappt. Der Ultraschall zeigt, was auch ohne Technik zu spüren war: Ein sehr großes Kind, Schätzgewicht $4500 \mathrm{~g}$, enormer Bauchumfang, sehr viel Fruchtwasser, eine sehr dicke Plazenta - alles Zeichen für einen unbehandelten Gestationsdiabetes.

„Wieviel haben Sie denn zugenommen?“ „30 Kilo, aber mein Arzt sagt, da ist auch viel Wasser dabei.“ „Haben Sie einen Zuckertest gemacht?" „Ja, der war ganz in Ordnung.“

Schneller Blick in den Mutterpass:

- Schwangerschaftsrisiken: „Oma Herzinfarkt“

- Ausgangsgewicht $78 \mathrm{~kg}$ bei $167 \mathrm{~cm}$, aktuell $109 \mathrm{~kg}$

- Beratung allgemein, z. B. Beruf, Reisen, Ernährung, Genussmittel, Sport angekreuzt.

- Spezielle Risikoberatung: angekreuzt.

„Hat Ihr Frauenarzt Sie über Ernährung beraten?“ „Ja, ich hab die Foliotabletten.“ „Haben Sie darüber gesprochen, dass Sie sehr viel zugenommen haben?“ „Nö.“

Aus dem Mutterpass purzeln diverse Quittungen:

„Schwangerschaftsbetreuung plus: Für zusätzliche, nicht durch die Krankenkasse finanzierte, aber empfohlene Untersuchungen wurden 231,80 Euro entrichtet.“

Geburtsvorbereitende Akupunktur, Cytomegalie-Test, Toxoplasmose-Test, 3D-Ultraschall - alles dabei.

Der „,kleine“ Zuckertest mit 50 g Glukose ergab einen Ein-Stunden-Wert von 135 mg\%, den Grenzwert, bei dem eine weitergehende Untersuchung empfohlen wird, die aber nicht durchgeführt wurde.

Die Schwangere hatte 10 Vorsorgeuntersuchungen, bei 9 wurde ein Ultraschall dokumentiert, zuletzt vor 2 Wochen.

Es wurden viele Bilder ausgedruckt, die Wachstumskurve ist jedoch nicht ausgefüllt.

Ich ärgere mich: Was ist das für eine Schwangerenbetreuung? Es wird untersucht und kassiert und dann wird die werdende Mutter 2 Wochen vor ET in die Klinik geschickt, wenn nichts mehr zu ändern ist!

Einleitung, wie soll das gehen? 2 Wochen vor ET bei einer übergewichtigen Erstgebärenden, die wegen des gestörten Stoffwechsels keine Prostaglandinrezeptoren ausgebildet hat. Und wenn es überhaupt so weit kommt, droht bei der Geburt eine Schulterdystokie. 


\section{Geschichtliche und gesell- schaftliche Entwicklung}

Glaubt man dem Berufsverband der Frauenärzte, ist das Problem keines:

„Mit den im Mutterpass aufgeführten Untersuchungen ist eine werdende Mutter rundum gut versorgt. Nur bei außerordentlichen medizinischen Verdachtsmomenten kann die ein oder andere $z u-$ sätzliche Vorsorgeuntersuchung sinnvoll sein. Zusätzliche Untersuchungen sollten in der Regel nach eingehender Prüfung und individueller Beratung durch die Frauenärztin bzw. den Frauenarzt erfolgen.“[1]

Das Medizinsystem ist nach dem Prinzip „Vergütung von Einzelleistungen“ aufgebaut. Man darf sich daher nicht wundern, wenn in einer Praxis nicht nur das medizinisch Sinnvolle, sondern auch (und manchmal leider ganz überwiegend) das finanziell Lukrative angeboten wird. In diesem Klima kann eine wertschätzende und salutogenetische Haltung nicht gut gedeihen.

1966 wurde in Westdeutschland die von den Krankenkassen finanzierte Schwangerenvorsorge eingeführt, nachdem die perinatale Mortalität mit 28/1000 weit über der von anderen europäischen Ländern lag.

Das Hickhack um Zuständigkeit und Vergütung der Schwangerenvorsorge begleitete bereits ihre Einführung. Die Idee, Vorsorge als Schwangerenberatung Hebammen und öffentlichen Institutionen wie Gesundheitsämtern zu übertragen, wurde schnell verworfen. Die Gesundheitsämter hatten in der Nazizeit eine unrühmliche Rolle gespielt. Gleichzeitig setzte sich die starke Lobby der niedergelassenen Ärzte für individuelle Untersuchungen in der Praxis ein. Sogar der Mutterpass als Dokumentation, die auch die Frauen und andere Personen einsehen können, wurde anfangs vehement bekämpft [4]: „...ist es uns jetzt gelungen, alle Bestrebungen nach Einführung eines Mutterpasses in Bayern zu unterbinden. Wir haben den Standpunkt vertreten, dass ärztliche Daten und Untersuchungsergebnisse in die Kartei des Arztes, aber nicht in die Handtasche der Frau gehören: Wenn sie in der Handtasche der Frau sind, werden sie Gegenstand von Unterhaltungen beim Kaffeeklatsch und führen zu einer unnötigen Beunruhigung der Frauen, die mit diesen nichts anzufangen wissen." H. J. Severing zitiert in [4]

„Weithin haben die Kassenärztlichen Vereinigungen es abgelehnt, Beratungsstellen in Kliniken an der Vergütung für Schwangeren-Vorsorge zu beteiligen. Die Folge: Wohlausgerüstete Beratungsstellen für Schwangere, die etwa an deutschen Universitätskliniken schon eingerichtet worden waren, mussten wieder geschlossen werden.“ [2]

Nach 1989 wiederholte sich diese Entwicklung in den neuen Bundesländern, wo gut funktionierende Ambulanzen und Polikliniken geschlossen wurden.

In der Folgezeit wurden die Untersuchungen ausgeweitet. 2 Ultraschalluntersuchungen wurden 1979 in den Katalog aufgenommen, 1995 kam eine 3. dazu.

Wenig bekannt ist bis heute, dass eine Hebamme die Schwangeren selbständig betreuen und sie zu den Ultraschalluntersuchungen an eine ärztliche Praxis überweisen kann.

Seit den 90er Jahren nimmt die Pränataldiagnostik einen immer größeren Raum ein. Ihre Ergebnisse führen selten $\mathrm{zu}$ einer besseren Gesundheit von Mutter und Kind, etwa wenn ein Herzfehler rechtzeitig entdeckt und die Operation schon vor der Geburt geplant werden kann. Meist bleibt nach einem auffälligen Ergebnis nur die Frage: Schwangerschaft fortsetzen oder abbrechen?

In den letzten 20 Jahren ist die perinatale Mortalität nur noch minimal gesunken, obwohl unzählige zusätzliche Untersuchungen angeboten werden.

Die geringe Reduktion ist teilweise darauf zurückzuführen, dass mehr Kinder nach einer frühen Frühgeburt überleben. Zum anderen werden viele Kinder, die früher kurz nach der Geburt an schweren Fehlbildungen gestorben sind, nicht geboren, weil die Schwangerschaft vorher abgebrochen wird. Die späten Schwangerschaftsabbrüche werden bis heute nicht systematisch erfasst, ihre genaue Zahl ist nicht bekannt.

Es gibt gute Übersichten darüber, welche Untersuchungen nach den strengen Kriterien der evidence based medicine sinnvoll sind (z.B. BzgA, Spiegel.de, Stiftung Warentest). Aber mit Vernunft allein lässt sich das Problem nicht lösen.

So wie beim Laufen an der frischen Luft nicht mehr das Gefühl der wohligen Erschöpfung anzeigt, dass es genug ist, sondern die Pulsmess-App, so möchte man sich auch in der Schwangerschaft apparativ absichern. Und wie Eckart von Hirschhausens Patient mit dem schmerzenden Knie gekränkt ist, wenn er beim Arzt nur beraten wird („Abnehmen und mehr Bewegung! Dafür zahle ich doch keine Beiträge!“), so beschweren sich auch Schwangere, wenn das, was gesundheitsökonomisch das beste Preis-Leistungs-Verhältnis hat, nämlich die sorgfältige Erhebung einer Anamnese, nicht durch weitere Aktionen ergänzt wird.

„war heute bei der Vorsorge und der hat nur sehr viel geredet und mich ausgefragt. Bild gab's auch net. Wie ist das bei euch, kriegt ihr immer ein Bild mit? Überlege, ob ich mein Gyn. wechseln soll“ Zitat von sannchen 84 bei www.netmoms.de.

Weil die Informationsmöglichkeiten nahezu unbegrenzt sind und Autonomie und Selbstverantwortung gefordert werden, ist die Verunsicherung sehr groß. Dies offenbaren die Beiträge in Schwangerschaftsforen. Daher wundert es nicht, dass die meisten Schwangeren die Ver- 
antwortung für ihre Schwangerschaft der Gynäkologin übertragen und die Betreuung durch Hebammen allenfalls als Ergänzung wahrnehmen.

Außerdem scheinen die Untersuchungen mit dem höchsten Erkenntniswert unattraktiv. Blutdruck messen, wiegen, Bauch abtasten ist im Vergleich zum 3DUltraschall langweilig.

Dazu kommt das für viele Frauen extrem belastende Thema „Gewicht“. Gerade für Frauen, die schon vor der Schwangerschaft permanent gegen ihr tatsächliches oder vermeintliches Übergewicht ankämpfen, ist das regelmäßige Wiegen vor Zeugen der schlimmste Teil der Untersuchung.

Bereits während meiner Doktorarbeit im ländlichen Westafrika 1989 faszinierte mich eine Untersuchung über die Schwangerenvorsorge in Tansania, die zeigte, dass Vorsorge die Gesundheit von Mutter und Kind zwar fördert, dass aber mehr als 4 Vorsorgeuntersuchungen keine Verbesserung brachten und dass die Entdeckungsrate der Präeklampsie in Deutschland und in Tansania bei je $80 \%$ lag. Ist mehr also nicht unbedingt besser?

Das ursprüngliche Ziel der Schwangerenvorsorge, möglichst viele Eklampsien zu verhindern, wurde durch einfache Untersuchungen wie die Blutdruckmessung, Gewichtskontrolle und Eiweißteststreifen bereits in den 1970er Jahren erreicht. Dagegen sind viele Probleme bis heute ungelöst, wie die Frühgeburtlichkeit.

Anstatt aber einen Blick auf die bekannten Risikofaktoren zu werfen (Armut, Rauchen, fehlende Partnerbeziehung, Gewalt), wird mit Technik gegengesteuert. Die vaginale Untersuchung ist nicht evidenzbasiert, wird aber trotzdem routinemäßig durchgeführt. Zusätzlich gibt es den vaginalen Ultraschall zur Messung der Zervixlänge und einen Teststreifen, der als Selbstzahlerleistung angeboten wird, eigentlich aber nur bei erhöhtem Frühgeburtsrisiko eingesetzt werden sollte.
„Ich habe die ganze Nacht nicht richtig geschlafen, meine FÄ hat mich gestern Abend angerufen und mir gesagt dass der Actim Partus test, den sie bei mir gemacht hat, (ist bei ihr Routine-Igel) leider positiv ist. Jetzt bin ich total verunsichert! Ich soll jetzt morgen nochmal reinkommen damit sie den Mumu kontrollieren kann." Zitat vonBeGe auf www.BabyCenter.de

Studiert man die Forenbeiträge, so macht sich das Gefühl breit, dass es in deutschen Praxen immense Kommunikationsdefizite gibt.

Wie bei allen Gesundheitsleistungen sind Frauen aus einkommensschwachen Verhältnissen unterrepräsentiert. Bis heute nehmen gut situierte Frauen durchschnittlich 2-3 Vorsorgeuntersuchungen mehr wahr. Präventionskampagnen, z.B. zum Rauchverzicht, sind an der Mittelschicht orientiert und erreichen die Zielgruppen häufig nicht [5]. Es fällt auf, dass das Risiko „besondere soziale Belastung“ in der Perinatalerhebung in keinem Bundesland über 1\% ausmacht, während bundesweit mittlerweile $15 \%$ der Bevölkerung als arm gelten [9]. Obwohl bekannt ist, dass Armut einer der Hauptrisikofaktoren für Frühgeburten, niedriges Geburtsgewicht und perinatale Mortalität ist, wird dieses Risiko offenbar in der Vorsorge nicht erfasst.

Auch hier finde ich übrigens eine Parallele zur Schwangerenvorsorge in Westafrika, zu der sich Funktionärsfrauen mit properen, wohlgenährten Kindern einfanden, während sich die Frauen aus dem Dorf aus Scham wegen ihrer Armut nicht trauten.

Dass mehr Vorsorge zu mehr Gesundheit führt, ist ein weit verbreiteter Irrtum. Viele Untersuchungen haben Ritualcharakter. Wie ich mich bei der Beichte von der Sünde befreie, so kaufe ich mich durch (im Einzelfall teuer bezahlte) Untersuchungen von Angst frei. Wenn dann noch ein gewisser Druck von Seiten der betreuenden Praxis kommt, eine Untersuchung durchzuführen, ist es aus mit dem informed consent.
„Ich würde gerne wissen, ob euch der Fa auch so eindringlich positiv berät wg. Selbtzahleruntersuchungen . Ich bin jetzt in der 9. Ssw und die meiste Zeit beim $\mathrm{Fa}$ geht für diese „Beratung“ drauf. Meine normalen Fragen zur Ssw, was ich beim Sport darf etc. werden mit einem 3-Wortsatz beantwortet. Bin ich nur extrem empfindlich oder ist das heutzutage normal beim Fa?" Zitat von musica13 auf www.BabyCenter.de

Es ist ein Mythos, dass die Schwangere wie eine Kundin zu Hause entspannt die Angebote studiert und sich nach ausgiebiger Information für das eine oder andere entscheidet.

\section{Individuelle Gesundheits- leistungen (IGeL)}

\section{Serologische Untersuchungen}

\section{Toxoplasmose}

Wenn bei der Mutter IgG-Antikörper nachgewiesen werden, kann sie sicher sein, dass sie bereits eine Toxoplasmose durchgemacht hat, die außerhalb der Schwangerschaft harmlos ist - wie ca. $50 \%$ aller Schwangeren. Falls keine Antikörper nachgewiesen werden, kann das zur Verunsicherung führen: Hat sie eine frische Infektion? Oder noch keinen Kontakt mit Toxoplasmose? Der Toxoplasmosetest ist ein gutes Beispiel dafür, wie ein Test weitere Tests nach sich zieht: er müsste die gesamte Schwangerschaft alle 4 Wochen wiederholt werden und würde trotzdem weiter zu Verunsicherung führen.

Dabei ist die Vorbeugung einfach: Eine Schwangere kann sich vor einer Infektion schützen, indem sie auf Hygiene beim Kontakt mit Katzen achtet und nur durchgegartes Fleisch isst.

\section{Der IGeL-Monitor rät daher vom Toxoplasmosetest ab.}

Auch beim Screening von anderen Infektionen bestehen große Unsicherheiten. Tests auf Cytomegalie und Ringelröteln 
beruhigen nur in den Fällen, in denen eine frühere Infektion bestätigt wird. Für alle übrigen Frauen, etwa die Hälfte, je nach Alter und Intensität des Kontakts mit kleinen Kindern, gibt es keine gute Lösung. Soll man während der gesamten Schwangerschaft regelmäßig kontrollieren? Inzwischen werden seronegative Schwangere schon fast routinemäßig aus der Arbeitswelt entfernt, wenn sie nur Kontakt mit Kindern haben.

Eine Lobby setzt sich in Deutschland für die Aufnahme des Cytomegalie-Screenings als Kassenleistungen ein mit dem Argument, dass seit einigen Jahren die Möglichkeit besteht, eine frische Infektion mit Hyperimmunglobulinen zu behandeln und so schwere Schäden beim Kind zu verhindern.

Gegen Windpocken sind über $90 \%$ aller Schwangeren immun, weil sie als Kind Windpocken hatten oder geimpft wurden. Theoretisch könnte man bei einer frischen Infektion Hyperimmunglobulin geben, da jedoch die Inkubationszeit lange ist und die Infektion fast immer zu spät festgestellt wird, gibt es in der Praxis keine Therapie. Sinnvoller als eine Serologie ist die Empfehlung, sich von kranken Kindern fernzuhalten.

\section{Ersttrimester-Screening}

Diese Untersuchung (Nackentransparenzmessung, Bestimmung von b-HCG und Papp-A) ist für viele Schwangere der niedrigschwellige Einstieg in die Pränataldiagnostik. Für viele Paare ist die Untersuchung (meist in einer Praxis für Pränataldiagnostik mit einem guten Ultraschallgerät) ein erstes Highlight. Die Untersuchung ist sinnvoll für Schwangere, die eine Entscheidungshilfe für oder gegen eine Amniozentese suchen. Es geht vornehmlich um die Suche nach der Trisomie 21.

Die Trisomien 13 und 18 sind sehr viel seltener. Da die Kinder meist schon während der Schwangerschaft intrauterin sterben, ist das ethische Dilemma
„Schwangerschaftsabbruch oder nicht?“ seltener als beim Down-Syndrom.

Über $90 \%$ der Schwangerschaften mit dem Befund Down-Syndrom werden inzwischen abgebrochen.

Das Problem sind auch hier unklare Befunde. Die Foren sind voll von Frauen, die verzweifeln, weil ihr Risiko von 1:700 auf $1: 382$ angestiegen ist. Menschen haben offenbar keine Möglichkeit, einen solchen Befund zu verarbeiten. Wie kann man individuell mit statistischen Wahrscheinlichkeiten umgehen?

„Diese ganzen Untersuchungen bringen den Ärzten nur Geld und machen dich wuschig. ...ich habe das über mich ergehen lassen aufgrund eines höchst verdächtigen Ultraschalls. Dann folgten die anderen Messungen (Nackenfalte 5,6 mm!!) Tripple, und und und. Wenn man bedenkt, dass zwischen diesen Untersuchungen jedesmal 1-2 Wochen lagen, war ein Drittel der SS nur mit Ängsten, Hoffen und Bangen versaut.“ Teetante4 auf GoFeminin.de

\section{Triple-Test}

Der Triple-Test (Bestimmung von Östriol, a-Fetoprotein und freiem b-HCG) in der 16.-18. SSW wurde ursprünglich als Screeningtest für ein erhöhtes DownSyndrom- und Spina-bifida-Risiko eingeführt. Er ist heute weitgehend durch das Ersttrimester-Screening ersetzt worden, das genauere Ergebnisse liefert und früher durchgeführt werden kann.

Die Spezifität des Triple-Tests ist sehr gering, d. h. er liefert viele falsch positive Ergebnisse und führt so zu großer Verunsicherung und sollte nicht mehr durchgeführt werden.

Trotzdem wird er noch in vielen Praxen angeboten.

\section{Screening auf b-Strepto- kokken}

Ein gutes Beispiel für ein sinnvolles Screening: Ein relativ sicherer Test auf eine Krankheit, die häufig ist und erfolgreich behandelt werden kann. Beim Nachweis von b-Streptokokken in den letzten Schwangerschaftswochen kann die werdende Mutter während der Geburt mit Antibiotika behandelt und so das Risiko einer gefährlichen Infektion des Neugeborenen deutlich vermindert werden. Die Untersuchung gehört in den USA zur regulären Schwangerenvorsorge. Auch in Deutschland wird ein generelles Screening aller Schwangeren empfohlen, bisher ist die Untersuchung aber keine Kassenleistung.

\section{Ultraschalluntersuchungen}

Die mit Abstand beliebteste Untersuchung ist der Ultraschall. In den Internet-Communities berichten Schwangere darüber, dass ihre Ärztin keine Bilder rausrückt, während andere schwärmen, bei ihnen gebe es jedes Mal eines in $3 \mathrm{D}$, „aber wir haben auch eine Flatrate gebucht für 180 Euro, das ist es uns wert“. Aber selbst scheinbar harmlose Vergnügungen wie der 3D-Ultraschall können verunsichern:

„...ich hatte gestern den $3 d$ ultraschall... es war echt voll toll wir haben sogar ein video wo der kleine drauf lächelt ... auf

\section{Fallbeispiel}

Frau H. hat nach der Einstellung auf Insulin noch eine Woche durchgehalten. Bei der geplanten Sectio wurde ein $4600 \mathrm{~g}$ schwerer Junge geboren, der normale Blutzuckerwerte hatte und nicht in die Kinderklinik verlegt werden musste wenigstens ein kleiner Erfolg. 
den bildern sieht es aus als hätte er eine ziemlich breite nase ... jetzt stellt sich mir aber irgendwie die frage ob das normal ist? also ob das am gerät liegt oder ist die nase wirklich so? was schlimmes kann es aber nicht sein oder?" Sunshine 2401 auf GoFeminin.de

Ob der Arzt eine Fehlbildung durch Ultraschall erkennt, hängt nicht von der Zahl der Untersuchungen ab, sondern von seiner Erfahrung [7]. Diese Binsenweisheit wird leider häufig ignoriert. Zum Glück ist hier die Statistik auf der Seite der Schwangeren. Die meisten Kinder sind unauffällig und sehen auch so aus, so dass die Bilanz positiv ausfällt.

\section{Fazit}

Für viele der IGeL ist (ebenso wie für einige kassenfinanzierte Untersuchungen) der Nutzen nicht nachgewiesen. Ob es heute noch sinnvoll ist, alle Schwangeren auf Syphilis zu testen, ist fraglich. Die routinemäßige vaginale Untersuchung schadet mehr als sie nutzt und ist vielen Frauen unangenehm. Wesentliche Schwangerschaftsrisiken werden oft trotz regelmäßiger aufwändiger Vorsorge nicht erfasst.

Der bürokratische Aufwand für Veränderungen der Mutterschaftsrichtlinien ist jedoch enorm und so wird es vermutlich noch viele Jahre dauern, bis aktuelle Empfehlungen umgesetzt werden. Das deutsche Medizinsystem bestraft finanziell das Gesunde und belohnt Pathologie. In der Gynäkologie profitiert man von Risikoschwangerschaften, weil mehr Untersuchungen abgerechnet werden können. Hier wünsche ich mir, dass Hebammen den vernachlässigten und von vielen Frauen sehnlich gewünschten Teil der Vorsorge übernehmen: durch Wahrnehmung der Schwangeren als Person und nicht als Ansammlung von Risiken Wertschätzung vermitteln, das Normale und Gesunde $\mathrm{zu}$ betonen und der Schwangeren im Dschungel von Fragen und Wahrscheinlichkeiten die gute Hoffnung zu vermitteln, die ihr die Kraft für ein gesundes Leben mit Kind gibt.

\section{Literatur}

1 Berufsverband der Frauenärzte, 2006: "Fact Sheet Schwangerenvorsorge“

2 „Mär von Karotten“, Der Spiegel 11/1968

3 Moller B1, Lushino O, Meirik O, GebreMedhin M, Lindmark G. Int J Gynaecol Obstet. 1989 Oct; 30 (2): 123-131.

A study of antenatal care at village level in rural Tanzania

4 Lengwiler M, Madarász J, 2014. Das präventive Selbst: Eine Kulturgeschichte moderner Gesundheitspolitik, Bielefeld

5 Lindner U, 2008. Präventionskonzepte im Umbruch: Schwangerenvorsorge in Großbritannien und Westdeutschland nach 1945. In: Tagungsbericht Transformation der Gesundheit zwischen Politik und Kultur 2008, Berlin

6 Deutscher Hebammenverband, 2014. Schwangerenvorsorge durch Hebammen, Hippokrates, Stuttgart

7 Focus Online: „Baby-Fernsehen“: Zusätzlicher Ultraschall während der Schwangerschaft http://www.focus.de/, abgerufen am 02.12.2014

8 http://www.igel-monitor.de/IGeL, abgerufen am 06.12.2014

9 Stiftung Warentest (Hrsg.), 2007: Untersuchungen zur Früherkennung. Für Schwangere. Nutzen und Risiken, Berlin
10 Bundesministerium für Arbeit und Soziales (Hrsg.): Lebenslagen in Deutschland - 4. Armuts- und Reichtumsbericht der Bundesregierung. 7. März 2013

11 Kurmann M, 2002. Zwischen Gewöhnung und Empörung. Pränataldiagnostik in der Schwangerenvorsorge Problemstellungen, Widersprüche, Aufklärung und Beratung. In: Brähler E, Stöbel-Richter Y, Hauffe U (Hrsg.): Vom Stammbaum zur Stammzelle.

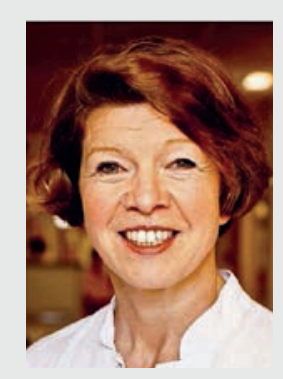

\section{Dr. Katharina Lüdemann} Chefärztin der Frauenklinik St. Josef-Stift, Westerstr. 10 27749 Delmenhorst E-Mail: Luedemann@sjs-del.de 